\title{
Scientific Legacy of the INCT-APA
}

\author{
Dr. Edson Rodrigues (UNITAU) - Vice-Team Leader of the Thematic Area 3 - \\ Impact of Human Activities on the Antarctic marine Environment, National Institute of Science \\ and Technology - Antarctic Environmental Research, INCT-APA
}

\author{
rodedson@gmail.com
}

https://doi.org/10.4322/apa.2016.021

The human presence and scientific activities developed in the Antarctica increased significantly during the International Geophysical Year of 1957/58 (IGY). More than a dozen scientific stations (McMurdo and AmundsenScott of the United States, Vostok of the former Soviet Union, among others) were constructed and about 5,000 people were displaced to the region between July 1957 and December 1958. Although the presence of humans substantially increased in the subsequent years, concern for environmental preservation became only evident in the 1970s, when the Antarctic Treaty consultative parties recognized the need to protect that region from human interference. Thus, in 1991, an ample protocol on environmental protection (Madrid Protocol) was approved, recommending the management of residues and emphasizing the importance of scientific research focused on environmental monitoring.

The National Institute of Science and Technology Antarctic Environmental Research (INCT-APA), created by the Ministry of Science, Technology and Innovation in 2009 , incorporated into its scope part of the guidelines of the Madrid Protocol and the IGY atmospheric sciences. Thus, studies on the physics of the ionosphere, meteorology, cosmic radiation, terrestrial magnetic field and monitoring of the ozone layer in Antarctica were carried out under the thematic area "Antarctic Atmosphere and Environmental Impacts in South America". The knowledge generated was essential to improve the understanding of the processes that operate in the terrestrial atmosphere, in regional and global scale.

The cooling and physical isolation of Antarctica about 30 million years ago led to the emergence of an endemic and stenothermic fauna adapted to low and stable temperatures in the marine environment. However, over the past 60 years, the accelerated warming of the Antarctic Peninsula and the increase in $\mathrm{CO} 2$ levels in the atmosphere has raised questions about the ability of acclimatization of Antarctic ectothermic organisms to this new environmental condition. The INCT-APA's "Impact of Global Changes on the Antarctic Terrestrial Environment" has studied the migratory behavior, feeding habits, and impacts of accelerated warming on Antarctic bird populations. Studies on flora have described new species in the Islands of the South Shetlands Archipelago, as well as have documented the presence of exotic species.

Studies carried out under the INCT-APA's "Impact of Human Activities on the Antarctic Marine Environment" showed that heating and low salinity are capable of altering the behavior of physiological systems of Antarctic ectotherms and compromising the life of these organisms in the long term. Chemical and microbiological markers were used to measure the extent of the polluting action of the Antarctic Comandante Ferraz Station (EACF) in the marine environment of the Admiralty Bay, showing that pollution reaches a few tens of meters. However, biochemical studies have shown that EACF sewage effluent is capable of raising the energy demand of Antarctic fish, as well as inducing metabolic alterations. Studies on bioaccumulation of oilderived hydrocarbons and organic pollutants in organisms from the Admiralty Bay trophic network have contributed to the monitoring of the continuing exposure of Antarctic biota to organic pollutants.

The extreme seasonality of the photoperiod and the extent of the layer of ice on the sea cause the biota to experience a long period of famine during the winter (about 8 months) and another one of feast during the austral summer. Trophic network studies carried out by the INCT-APA comprise organisms from the bottom of the food 
chain to top predators. The majority of these studies were conducted in the Specially Managed Area of Admiralty Bay (ASMA \# 1), King George Island, located at the northern tip of the Antarctic Peninsula, which is undergoing accelerated warming.

The leaching in volcanic soil of the Antarctic Peninsula and adjacent islands during the Southern summer has been pointed out as the main factor responsible for the naturally high levels of heavy metals found in intertidal and infralittoral organisms. Researchers of the INCT-APA have carried out baseline studies to monitor the levels of heavy metals in the soil and sediments of ASMA \# 1 in Admiralty Bay. On the other hand, a study conducted with Nacella concinna showed that the exposure of this gastropod to the naturally high levels of copper over an evolutionary time scale could have resulted a metabolic microevolution that provided advantage in the occupation of copper-rich niches. 